\title{
Science didactique et réputation littéraire : le sujet en question
}

Didactic science and literary reputation: the subject in question

\section{Yann Vuillet et Bruno Védrines}

\section{(2) OpenEdition}

\section{Journals}

\section{Édition électronique}

URL : https://journals.openedition.org/pratiques/9609

DOI : 10.4000/pratiques.9609

ISSN : 2425-2042

Éditeur

Centre de recherche sur les médiations (CREM)

Référence électronique

Yann Vuillet et Bruno Védrines, « Science didactique et réputation littéraire : le sujet en question », Pratiques [En ligne], 189-190 | 2021, mis en ligne le 09 juillet 2021, consulté le 23 juillet 2021. URL http://journals.openedition.org/pratiques/9609; DOI : https://doi.org/10.4000/pratiques.9609

Ce document a été généré automatiquement le 23 juillet 2021.

(c) Tous droits réservés 


\title{
Science didactique et réputation littéraire : le sujet en question
}

Didactic science and literary reputation: the subject in question

\author{
Yann Vuillet et Bruno Védrines
}

\begin{abstract}
Notre article, de nature théorique, propose une analyse compréhensive et critique de la rencontre de la didactique et de la littérature sous l'angle des processus par lesquels s'opère la subjectivation des individus, c'est-à-dire leur transformation en sujets. Pour aborder ces questions inévitablement polémiques, nous nous plaçons dans la continuité de travaux signalant d'une part l'absence d'extériorité des sciences sociales aux objets qui les intéressent (Adorno, 2016 ; Hacking, 2008 ; Passeron, 2006 ; Grignon \& Passeron, 2015), et inscrivant d'autre part la discipline «didactique du français » au sein du champ pluridisciplinaire des sciences de l'éducation (Hofstetter \& Schneuwly, 2007; Bronckart, 2019). Nous étudions les implications du triple problème conceptuel du didactique, du littéraire et du sujet, chacun des référents auxquels renvoient ces termes étant en effet pluriel, et discutable.
\end{abstract}

\section{L'espace sémantique de la didactique est celui des sciences sociohistoriques}

2 Avec son épistémologie descriptive, J.-C. Passeron oppose aux définitions à priori et abstraites de la science une organisation fondée sur la pluralité de ses «espaces assertoriques » (2001, p. 34). En voici une schématisation possible : 
Fig. 1 : Schématisation des sciences en fonction de leurs différents espaces assertoriques ou sémantiques, d'après les propositions de Passeron (2001).

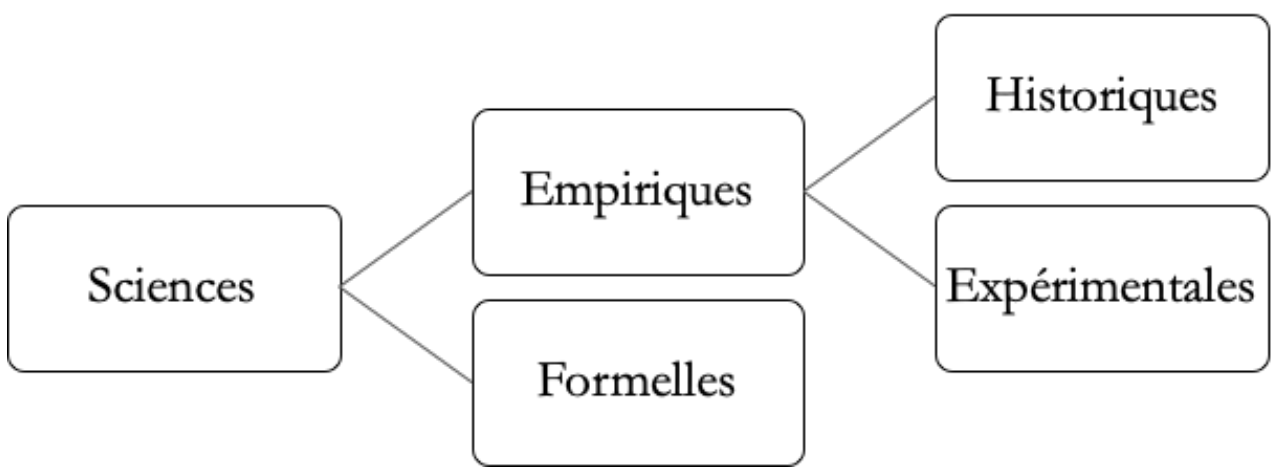

3 Tandis que les sciences formelles se concentrent sur l'administration de preuves de type logico-mathématique, les espaces assertoriques ou sémantiques des sciences empiriques dépendent nécessairement de la mise en correspondance de discours (de modèles, de concepts...) avec une variété de référents empiriques au travers de dispositifs de recherche adaptés (méthodes, instruments...). Deux espaces assertoriques ou sémantiques peuvent être en outre distingués au sein des sciences empiriques. Du côté tout d'abord des sciences expérimentales, les démonstrations réfutables (Popper, 1985) qui s'y administrent peuvent être vues comme s'inscrivant au sein de "paradigmes » (Kuhn, 1983) où s'exhibent discursivement de légitimes prétentions à l'universalité. Du côté des sciences historiques en revanche - dont la didactique telle que nous proposons de l'entendre -, l'historicité des objets oriente de façon différente le rapport de référence entre les théories, les modèles, les discours et l'empirie (Passeron, 2001, p. 35). Ces sciences ne sauraient fonctionner, comme c'est le cas des sciences expérimentales, par l'intermédiaire de la formulation et de la vérification de "modèles universels » des phénomènes empiriques approchés. Elles dépendent pour leur part, inévitablement, de "modèles à déictiques » (Passeron, 2001, p. 49), c'est-à-dire "de modèles qui exigent, pour prendre un sens explicatif, que leur soient associées des descriptions faisant intervenir d'autres informations que celles qui sont simplifiées et sélectionnées pour les besoins de la modélisation" (Passeron, 2001, p. 51). Les informations en question, non explicitement retenues par les modèles et néanmoins nécessaires à leur activation, peuvent en effet être pensées et décrites comme impliquant des déictiques : elles renvoient à un "ici » et à un «maintenant", à des interactions entre individus singuliers, à des conditions qui, parce qu'elles se matérialisent de manière toujours spécifique, ne peuvent pas se reproduire à l'identique dans des situations ultérieures ${ }^{1}$. Nous verrons que notre compréhension de la subjectivation permet de tenir compte de ce paramètre essentiel pour le raisonnement didactique.

\section{Les modèles de la didactique}

4 Comme toute science sociale, la didactique évolue au travers d'une double historicité : celle de ses objets, bien sûr, mais aussi celle des modèles qu'elle conçoit et active pour sélectionner ces mêmes objets, les décrire, les expliquer ou les critiquer. Leur histoire en témoigne, les modèles conçus par, et activés dans la science didactique sont fréquemment débattus, amendés, affinés, reformulés ou étendus en fonction des cas 
approchés comme en fonction des orientations choisies pour une recherche. Or l'évolutivité et la multiplicité des modèles ne reflètent pas un manque de formalisation provisoire que pourrait venir combler une théorie générale à venir. Elles constituent plutôt une condition structurelle de ce champ. Parce que la didactique procède à travers la modélisation de systèmes sociaux - à l'exemple du "système didactique », dont on peut d'ailleurs rappeler avec B. Daunay (2013) qu'il reste théoriquement peu construit il demeure toujours, dans les raisonnements des didacticiens comme dans ceux des sociologues, un «extérieur (...) qui reste heuristiquement inséparable de l'objet » qu'ils construisent (Passeron, 1994, p. 102). En didactique, cet extérieur désignera ce qu'une théorie, un modèle, un concept ne retiennent pas dans une forme donnée, mais qui pourrait, en fonction des argumentations, des apports pour une analyse scientifique ultérieure, en fonction des dimensions rendues observables par un ensemble de cas, être intégré ou pris en compte avec plus de systématicité dans un nouveau raisonnement didactique. Partant, à moins de refouler la double historicité des objets et des modèles hors des processus de construction et de description des phénomènes touchant à l'enseignement et à l'apprentissage de savoirs, il doit être admis que la didactique, lorsqu'elle s'intéresse à ce qui se passe empiriquement dans des classes ordinaires, produit bien des interprétations ${ }^{2}$ qui ne sauraient être (in)validées sur un plan formel ou strictement expérimental.

Dans ces conditions, les modélisations des objets sociaux approchés prennent des formes notablement diverses. Par exemple et pour n'évoquer qu'eux, de la didactique du curriculum (Lebeaume, 2011; Martinand, 2016) à l'action conjointe (Sensevy \& Mercier, 2007), de la théorie de la transposition (Chevallard, 1994 ; Schneuwly, 1995) à celle des situations (Brousseau, 1998), de nombreux modèles peuvent être activés pour décrire des phénomènes d'enseignement et d'apprentissage. Sans les épuiser, en fonction des disciplines, des courants qui s'y développent et bien sûr en fonction des objets d'enseignement et d'apprentissage, les différences entre les modèles attestables en didactique portent sur des choix et des orientations liées aux variables suivantes :

\begin{tabular}{|l|l|}
\hline Variables & Exemples \\
\hline Échelle spatiale & Espace géographique : pays, région, ville ou localité... \\
\hline Échelle temporelle & $\begin{array}{l}\text { Histoire de l'enseignement d'une discipline, de ses curricula, séance } \\
\text { d'enseignement, évènement remarquable... }\end{array}$ \\
\hline $\begin{array}{l}\text { Dimensions } \\
\text { épistémologiques } \\
\text { praxéologiques }\end{array}$ & $\begin{array}{l}\text { Modélisation des objets d'enseignements : prise en compte (de la } \\
\text { transformation) de savoirs savants, de savoirs d'experts, de pratiques } \\
\text { sociales de référence; attention portée aux élaborations didactiques des } \\
\text { savoirs... }\end{array}$ \\
\hline $\begin{array}{l}\text { Théories psychologiques } \\
\text { de référence }\end{array}$ & $\begin{array}{l}\text { Psychologie génétique piagétienne, théorie des champs conceptuels, } \\
\text { psychologie développementale vygotskienne... }\end{array}$ \\
\hline $\begin{array}{l}\text { Modèles d'analyse de } \\
\text { l'activité }\end{array}$ & $\begin{array}{l}\text { Théorie des jeux, agir communicationnel et praxéologique, médiation, } \\
\text { action conjointe, clinique de l'activité... }\end{array}$ \\
\hline Dispositifs didactiques & $\begin{array}{l}\text { Situations-problèmes, travail par problématisation, problèmes ouverts, } \\
\text { séquences didactiques, situations adidactiques, questions socialement } \\
\text { vives... }\end{array}$ \\
\hline Conceptions du « sujet » & \begin{tabular}{l} 
Épistémique, socio-affectif, didactique... \\
\hline
\end{tabular} \\
\hline
\end{tabular}




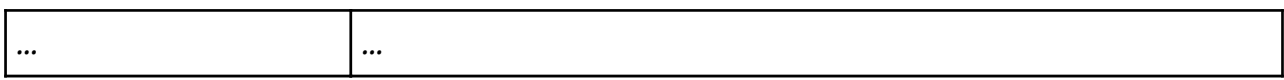

6 Cette diversité n'est pas à déplorer: considérer que la didactique est une science sociohistorique permet d'accepter qu'une grande variété de modèles concurrents - ou complémentaires - puisse y coexister sans que l'absence d'un paradigme unifié n'affecte la légitimité ni l'intérêt d'aucun d'entre eux. Simultanément, le fonctionnement de l'espace sémantique de la science didactique légitime la reprise, la reformulation, l'extension de ses modèles existants, de même qu'elle autorise la critique de leurs possibles réductionnismes. Or c'est précisément de cette marge de manœuvre que nous profitons pour (ré)interroger les processus de transformation d'individus en sujets didactiques. A cet effet, nous apportons encore quelques éclairages préalables sur la construction scientifique de l'empirie.

\section{Les dimensions pratiques et éthiques de la construction de l'empirie didactique}

7 Construire une empirie engage une conception « du » didactique, soit un modèle sans lequel il serait impossible de délimiter quelque objet didactique que ce soit au sein de l'ensemble des phénomènes sociaux. Or la vivacité des débats entourant «le » didactique, dont témoigne par exemple le numéro 8-1 de la revue Éducation et didactique (2014), suggère de rester ouvert à une conception relativement large, mais néanmoins assez précise et explicite pour ouvrir à la critique et à la remise en question. Inspirés par J.-L. Martinand (2014, p.360), nous admettrons pour notre part que " du» didactique se manifeste à chaque fois que s'exerce une forme de responsabilité vis-à-vis de contenus d'enseignement et d'apprentissage.

Cependant, Il ne suffit pas d'avancer que l'empirie didactique peut être constituée par l'ensemble, sécable, des processus qui concourent à l'exercice de responsabilités vis-àvis de contenus d'enseignement et d'apprentissage. Encore nous semble-t-il important de préciser, toujours en nous inspirant de J.-L. Martinand (2014, p.360), que ces responsabilités s'inscrivent dans des espaces d'activités variés - prescription, enseignement et apprentissage, recherche, formation - dont les enjeux respectifs viennent spécifier des orientations différentes, pouvant être qualifiées de normative ou interventionniste (prescrire des enseignements et des apprentissages), pragmatique (réaliser des enseignements et des apprentissages) ou encore descriptive ou critique (décrire et critiquer des phénomènes didactiques). À travers cette perspective relativement large, l'empirie didactique apparait donc comme un assemblage d'espaces, bénéficiant chacun d'une autonomie relative, où trouvent à se loger des orientations, des manières hétérogènes de penser, de dire et d'agir.

Si l'on porte crédit aux propositions qui précèdent, il est encore nécessaire de prendre en compte les implications cruciales, de nature pratique et éthique, nécessairement liées à l'espace sémantique décrit ci-dessus comme étant celui de la science didactique. Voudrait-elle s'en tenir à une position neutre et purement descriptive vis-à-vis des situations d'enseignement et d'apprentissage et des sujets y évoluant? La didactique ne le pourrait pas, ne serait-ce qu'en raison de l'absence d'extériorité qui caractérise le rapport entre les sciences sociohistoriques et les objets sur lesquels elles font porter leur attention. Sur ce plan général, en effet, il n'est pas inutile de rappeler que le 
discours le plus descriptif qui soit, lorsqu'il porte sur des êtres humains (sur leurs actions individuelles, sur leurs activités sociales), est susceptible de les transformer s'ils apprennent ce qui est dit d'eux (Hacking, 2008).

L'omniprésence virtuelle ou effective des aspects pragmatiques de la science didactique nous mène enfin à pointer que son potentiel transformatif ne se limite pas aux seuls contenus. Sa dimension «théorico-pratique » (Halté, 1992, p. 17) engage simultanément des responsabilités éthiques vis-à-vis de la transformation de personnes en sujets dont les activités revêtent un caractère didactique - au premier rang desquels, bien sûr, se trouvent les apprenants... et les enseignants. Tout en faisant l'hypothèse que les assujettissements ${ }^{3}$ se déclinent en fonction de chaque discipline, nous souhaitons pour notre part contribuer aux réflexions sur les "sujets didactiques" (Chevallard, 1988, 2010 ; Schubauer-Leoni, 1997 ; Daunay, 2007, 2016) en mettant notamment en évidence que la focale disciplinaire doit être spécifiée à un fin degré d'analyse afin de pouvoir rendre compte de cet aspect de l'empirie. En effet, vouloir décrire les processus concrets d'assujettissement didactique nous semble requérir de s'intéresser aux systèmes, toujours singuliers, que forment les relations entre : 1 ) les objets enseignés eux-mêmes référés ou référables à des théories ou à des pratiques sociales diverses, voir concurrentes; 2) les tâches organisées au sein de dispositifs; 3) les activités effectuées par les enseignants ; 4) les activités effectuées par les apprenants.

\section{La construction scientifique de modèles en didactique du français et la « réputation littéraire »}

Pour aborder les questions vives qui entourent les approches didactiques de la littérature, nous soulignons tout d'abord qu'une empirie didactique, quelle qu'elle soit, est inséparable d'un positionnement sur un axe générique-spécifique (Sensevy \& Ligozat, 2017). Cela est dû à cette «structure fractale» que Y. Chevallard signale « aux différents niveaux d'analyse du didactique » $(1998$, p. 106) et qui organise pour partie les relations entre les didactiques disciplinaires (celle du «français» est une spécification disciplinaire "du» didactique parmi d'autres) aussi bien qu'elle peut s'observer au sein même de chaque didactique (par exemple, celle de la production écrite est une spécification de la didactique du français, tout en demeurant dans un rapport de généricité vis-à-vis de l'approche de la production écrite de textes narratifs littéraires).

Dans le cadre de la discipline "français ", et ce phénomène a été largement analysé ${ }^{4}$, le positionnement relatif sur l'axe générique-spécifique s'accompagne d'une sélection de modèles, issus de disciplines contributrices ou créés au sein de la didactique du français, qui servent à approcher les objets sociohistoriques constituant la discipline. Alors que la "question des savoirs" à enseigner est à elle seule "nécessairement polémique et dépend de l'institution depuis laquelle elle se formule » (Chevallard, 1994, p. 172), nous remarquons qu'il en va de même sur le plan des théories de référence mises à profit pour décrire les processus d'enseignement et d'apprentissage. Or cette hétérogénéité théorique complexifie fortement la construction scientifique de la didactique du français dans son ensemble. Ce problème, qui peut être tenu pour indépassable, se manifeste d'une façon particulière à l'endroit des approches de la littérature. 

l'apprentissage de la littérature, la didactique du français se trouve confrontée à l'impossibilité de décrire de façon universelle ce qui fait qu'un texte, une activité lectorale ou scripturale ou une institution (entre autres) peuvent être reconnus ou non comme " littéraires ${ }^{5}$. Elle tend dès lors à renoncer au questionnement théorique pour préférer des discours plus praxéologiques - à l'exemple de ce que B. Louichon (2011) pointe à l'endroit de la « lecture littéraire » (p. 208). Or nous avons montré ailleurs que cette absence d'univocité provient du fait que le littéraire est une valeur qui déclenche des attributions, toujours contestables, de "réputation littéraire $»^{6}$ (Vuillet, 2017; Védrines, 2017; Vuillet \& Gabathuler, 2017; Védrines \& Gabathuler, 2018; Vuillet, 2018 ; Gabathuler et al., 2019).

la " dichotomie implicite entre "science des objets" et "science des sujets" " (Ligozat et al., 2014, p. 107) qui s'observe parfois en didactique. À cet effet, parce que les valeurs constitutives des objets littéraires sont les produits d'activités sociales contextualisées et parce que ces activités requièrent des individus qu'ils agissent et interagissent de manières spécifiques, il apparait nécessaire de pouvoir s'appuyer sur une théorie du «sujet » opératoire en didactique. De cette façon, il devient notamment possible de modéliser et de décrire certains phénomènes didactiques liés à la réputation littéraire.

\section{Que signifie être sujet dans un système didactique?}

15 La question du sujet didactique ne peut faire l'économie d'une réflexion sur ce que l'on entend par subjectivité et de son rapport avec une identité présumée. La manière de comprendre ce rapport parcourt le panorama des conceptions du sujet, de la plus idéaliste à sa mise en cause la plus radicale. Or, même si de nombreux arguments critiques ont amené à douter de l'existence d'un sujet autonome, souverain et libre ${ }^{7}$, nous n'en restons pas moins confrontés aux diverses idéologies du sujet qui affectent nos existences. D'ailleurs, comment serait-il envisageable dans notre environnement social contemporain de ne pas nous comporter selon les normes requises pour être reconnus en tant que sujet - «interpelé », pour reprendre le vocabulaire de L. Althusser (2011), en tant que sujet? Comment serait-il possible de ne pas agir en conséquence? de ne pas éprouver le sentiment d'avoir une subjectivité, ou du moins d'être socialement assigné à l'éprouver, la manifester, en rendre compte?

La définition que propose E. Balibar de l'idéologie comme " forme de conscience et d'inconscience (de reconnaissance et de méconnaissance) dans laquelle les individus vivent imaginairement leur rapport à leurs conditions d'existence » (2005, p. X) évite de ranger l'imaginaire idéologique systématiquement du côté de l'erreur - antithèse de la science. Si elle n'est qu'illusion, en effet, il s'avère alors difficile, comme le fait remarquer L. Althusser, d'expliquer son efficacité réelle dans les rapports sociaux. Il faut bien qu'il y ait un certain intérêt social objectif à persévérer dans ce qui peut apparaitre d'un autre point de vue comme une erreur (2014, p. 259). Dans cette perspective, un contexte social conflictuel ou antagoniste demande de comprendre pourquoi un individu tient à certaines formes d'assujettissement et si un renoncement ne représente pas pour lui un cout trop important en termes d'identité, voire un danger pour la cohérence de sa personnalité. Combien de jugements portés du point de vue du maitre ont-ils 
disqualifié les décisions et les comportements d'élèves parce qu'ils n'obéissaient pas à la «bonne » rationalité ?

L. Althusser en vient ainsi à proposer une théorie de l'identité en lien avec l'idéologie utile pour une meilleure compréhension du sujet didactique. À la question de savoir pourquoi il faut accepter les termes dans lesquels notre «je » est pressenti, appelé, interpelé, L. Althusser répond : c'est parce que le sujet idéologique - de même que le sujet didactique qui en constitue une forme de spécification - est antérieur à l'existence de l'individu, et donc que l'existence en tant que sujet ne peut s'effectuer que dans les termes de l'interpellation; c'est ce qu'il appelle " les rituels de la reconnaissance idéologique, qui nous garantissent que nous sommes bel et bien des sujets concrets, individuels, inconfondables et naturellement irremplaçables » (2011, p. 296).

Dans un cadre scolaire, nous dirons que la cohabitation et la hiérarchie des sujets dans un même élève dépendent certes des assujettissements hors scolaires, mais aussi et fondamentalement des disciplines et de leurs dispositifs didactiques. En faisant entrer les élèves dans des manières de dire, de penser et d'agir disciplinaires, autrement dit en mettant en œuvre leurs processus de « disciplination » (Hofstetter \& Schneuwly, 2014), les disciplines déclenchent des variations dans les formes d'assujettissement imposées aux élèves. D'ailleurs, ce phénomène peut entrainer des conflits intérieurs avec le horsscolaire, lorsque les processus de disciplination en biologie ou en histoire, par exemple, s'opposent aux croyances religieuses enseignées en d'autres lieux. On peut ainsi se demander si nombre de confusions et de malentendus qui désorientent les élèves ne proviennent pas de formes d'assujettissements qu'il serait utile de savoir catégoriser, associer, dissocier ou hiérarchiser.

\section{Les formes de sujet dans le système didactique}

Pour questionner cette conscience familière et quotidienne d' "être un sujet ", mais aussi pour éviter de la présenter comme point de départ et centre à partir duquel tout se fédère et prend sens, nous proposons de considérer le système didactique ${ }^{8}$ à partir des formes de sujet mises en œuvre en son sein, en fonction de la double variabilité des contenus et des tâches qui participent à la délimitation des savoirs dits littéraires et, plus largement, à l'évolution de la discipline scolaire « français ».

La réflexion didactique sur le sujet peut se développer selon des axes divers: personnaliste, épistémique, psychologique, psychanalytique, sociologique ou à l'inverse adopter une méfiance suspicieuse à l'égard de sa longue histoire idéaliste, préférant par crainte de multiples ambigüités et malentendus ne pas l'utiliser' ${ }^{9}$. La difficulté, dans tous les cas, est de prendre en compte un système didactique dynamisé par ses diverses composantes. C'est pourquoi le concept de sujet didactique nous parait essentiel, à condition toutefois de ne pas renoncer à une analyse critique du sujet avant même d'interroger ce que la didactique peut en faire ${ }^{10}$.

\section{Les sujets didactiques}

21 Dans une classe, le sujet didactique apprenant apparait dans sa relation avec l'enseignant aussi bien qu'avec les autres élèves, et ces relations sont médiées par l'objet d'enseignement ainsi que par le dispositif didactique. Aussi, dans une telle situation, décrire l'expression d'une subjectivité singulière et autonome - un sujet 
originel qui entrerait en formation, une sorte de tabula rasa de la subjectivité, point de départ sur lequel viendraient s'empiler les enseignements -, nous parait aussi abstrait et artificiel que le prétendu accueil de toutes les particularités des différents sujets sociaux agrégés dans un individu.

D'ailleurs, comment démontrer rationnellement qu'un élève serait plus sujet qu'un autre, c'est-à-dire plus proche de sa subjectivité propre et authentique qu'un autre ? En fonction de quels critères ? Parce qu'il serait plus fidèle à l'expression de ce qui, étant sa « vraie " subjectivité, correspondrait le mieux à sa "vraie " personnalité ? Mais s'agirait-il là d'un enseignement? En plus d'entrainer des jugements sur la sincérité, la sensibilité, l'intelligence, cette appréciation risque fort d'être relative à l'image que l'observateur se fait de la subjectivité authentique et des diverses idéologies du sujet qu'il porte, défend ou cherche à promouvoir ${ }^{11}$. En fait, il est contradictoire d'espérer trouver un autre sujet (qui serait plus vrai que le sujet scolaire) dans un milieu scolaire. L'individu étant fragmenté en sujets, on peut parler d'injonction contradictoire quand, dans un milieu didactique, on appelle un autre sujet que celui que le dispositif interpelle comme apprenant. Dans un système didactique, parce qu'il est didactique, on ne trouvera jamais que du sujet didactique, même si l'on prétend parfois que c'est le sujet authentique que l'on veut entendre.

Sous l'effet de la transposition interne, les sujets externes à l'école ne sont pas sollicités en tant que tels, mais se trouvent plutôt eux-mêmes transposés dans le cadre d'une tâche, d'un dispositif vecteur d'activités, à l'intérieur d'un système didactique orienté vers un objet de savoir "à savoir " (Schneuwly, 1994); c'est par exemple une narration à rédiger, au passé, sur ses propres vacances d'été. Et si l'expression de la subjectivité ne convient pas, dans ce dispositif, c'est parce qu'elle est considérée comme parasitant le système, et la raison n'est pas que l'école serait aliénante et coercitive, mais qu'elle est moment et lieu de formation. En d'autres termes, dans un système didactique, il y a des places prévues pour certains types de sujets, des impossibilités de sujets, et des transformations de sujets. Cela ne veut pas dire que ces sujets ne peuvent pas s'exprimer (tout le monde sait que les élèves ne s'en privent pas, compromettant même, à l'occasion, la possibilité de transmission « du » savoir), mais cette expressionlà n'est pas reconnue, valorisée.

Cette analyse doit s'étendre aux sujets didactiques enseignants. Certes, les enseignants sont les garants du savoir, mais ils n'en demeurent pas moins pris dans les processus dynamiques des tâches et des activités. Il leur arrive ainsi de devoir répondre à l'injonction institutionnelle de travailler en classe avec des objets réputés littéraires qui les laissent eux-mêmes perplexes (Vuillet \& Gabathuler, 2017). Ils mettent en place et régulent en continu les dispositifs didactiques permettant de s'approcher des apprentissages prescrits, tout en composant avec des nouds idéologiques qui surviennent (Vuillet, 2017). Ils déclenchent ou valident le passage à une nouvelle tâche. Ils prennent connaissance et accompagnent les activités de leurs élèves. Ils sont susceptibles d'entrer en empathie ou de maintenir une plus grande distance lorsque ces attitudes peuvent soutenir les apprentissages visés. Ils peuvent changer de position (magistrale, accompagnatrice, retrait...) dans les interactions en fonction des moments de la construction de l'objet enseigné. Autrement dit, les enseignants sont assujettis par la pratique de l'enseignement, elle-même concomitante à l'assujettissement didactique des apprenants, et sont constamment transformés par elle. 


\section{L'objet enseigné et la formation de types de sujets}

du troisième pôle, dans notre perspective, il s'agit avant tout aux objets d'enseignement dans la mesure où ils supposent le déploiement de dispositifs didactiques qui, tout en portant sur des contenus particuliers, impliquent des activités contribuant à la formation de types de sujets. Ce n'est donc pas l'objet à enseigner espéré par les plans d'étude qui assujettit véritablement, ni même les savoirs des savants, ceux des experts, ou plus largement les pratiques sociales prises en référence par les systèmes éducatifs. L'objet réellement enseigné : voilà ce qui assujettit pratiquement, non pas que les phénomènes de transposition externe n'aient pas d'importance ou d'influence - loin de là, mais ils ne forment pas, en tant que tels, l'empirie du processus d'assujettissement opérant en classe. De notre point de vue, ils orientent la mise en œuvre d'un projet d'assujettissement et préparent des situations didactiques pour les sujets, mais la réalisation dépend d'autres facteurs.

L'objet enseigné permet ainsi de réfléchir à la généalogie des objets (historique et idéologique), dans la mesure où ils sont une matérialisation des possibles, ce qui permet de rendre compte des potentialités qu'ils ouvrent, mais aussi de celles qu'ils empêchent. La question se pose nécessairement par exemple pour le choix des corpus de textes, des exercices scolaires, des dispositifs, des tâches, etc. L'explication de texte, par exemple, est un exercice qui permet d'observer à la fois un acte fait à l'intérieur d'une collectivité selon des règles précises d'énonciation, et en même temps le fait que cet acte - par l'autoréférence - est un énoncé assumé par un individu, et donc une manifestation de son assujettissement. Il est nécessaire d'insister sur la médiation de cette technique qu'est l'exercice, de sa pratique qui a une longue histoire et qui permet de mieux appréhender le fonctionnement de la discipline à l'origine du sujet didactique littéraire. Ainsi, pour un élève, le dispositif de l'explication de texte signifie certes en adopter la méthode, mais cette adhésion engage dans le même mouvement une construction spécifique du sujet, et qui ne peut être atteinte dans cette forme que par le dispositif en question. Dès lors, si l'on pose le problème à partir de l'assujettissement par le dispositif, on constate que les prises de position sur les méthodes d'explication de texte provoquées par les réformes successives génèrent inévitablement des conceptions variables du sujet et de sa formation sensible.

\section{Un exemple d'assujettissement : le style réputé littéraire}

Les transcriptions de leçons à partir de recherches portant sur l'enseignement littéraire (Gabathuler, 2016, Fallenbacher-Clavien, 2017, Védrines, 2017, Ronveaux \& Schneuwly, 2018) font souvent mention du style. Ce n'est guère étonnant puisque le style, héritier d'une longue tradition d'exégèse, en est venu à être une dimension essentielle de l'explication de texte littéraire et occupe une grande partie des efforts des élèves et des enseignants.

D’après C. Noille-Clauzade, la valorisation du style, sur le versant de l'histoire littéraire institutionnalisé dans l'enseignement par l'explication de texte, doit beaucoup à G. Lanson. En s'inspirant de l'exégèse théologique, et en particulier des quatre sens du texte biblique - littéral, allégorique, moral et anagogique - G. Lanson octroie au texte une profondeur de sens exceptionnelle et en fait, puisqu'il est commenté dans ce lieu public qu'est la classe, un objet commun d'admiration : « en littérature la religion des 
textes se parachève en une mystique du style » (2004, p. 25). C. Noille-Clauzade ajoute que la force du modèle tient à son institutionnalisation. Dominant, il va se perpétuer dans les études de lettres, devenir la référence majeure à partir de laquelle les autres théories du style devront se définir.

Dans notre perspective qui vise à déceler les traces d'une idéologie littéraire et la forme de subjectivité qui en découle, il est également intéressant de relever l'influence du romantisme et la dimension théologique que souligne L. Spitzer: "Ce n'est pas un hasard si "le cercle philologique" a été découvert par un théologien, qui avait pour tâche de rendre son harmonie à la discorde, et de restituer à ce monde la beauté de Dieu. Cette attitude se manifeste dans le mot fabriqué par Schleiermacher: Weltanschauung: Die Welt anschauen, voir, connaitre le monde jusque dans son détail sensible» $(1970$, p. 65). La cohérence est assez remarquable pour être soulignée: l'œuvre relève ici d'une totalité organique qu'il s'agit de restituer à partir de faits linguistiques pour accéder à la conscience d'un auteur qui, lui-même, prend place dans une mentalité collective. L'arrière-plan mystique du style et du littéraire parait patent.

Précisons encore que la distinction entre théorisation herméneutique et théorisation rhétorique proposée par C. Noille-Clauzade s'avère particulièrement intéressante, puisque les deux orientations se retrouvent dans les observations de leçons. La conséquence en est deux assujettissements à deux subjectivités bien distinctes. Sans trop entrer dans les détails, nous pouvons dire que cohabitent dans les classes d'aujourd'hui une conception du littéraire héritée de l'herméneutique dont la mystique porte sur la "personnalité » stylistique de l'auteur, à travers une vision du monde incarnée dans la facture de la langue, et parallèlement une conception rhétorique de tradition très ancienne, avec sa logique et ses outils spécifiques. C'est d'ailleurs cette dernière qui se régénèrera $\mathrm{au} \mathrm{xx}^{\mathrm{e}}$ siècle dans la stylistique, cette fois comprise comme un domaine linguistique. Peu importe au fond les controverses portant sur l'étendue de son champ théorique : ce qui semble plus significatif, c'est qu'avec la stylistique «nous sommes plus près de la pensée rhétorique des styles comme registres de procédures formelles", tandis que "c'est dans ce cadre linguistique général que la théorie contemporaine a tenté de faire du style un concept permettant de penser la notion de "littérarité" » (Noille-Clauzade, 2004, p. 40). Or, c'est sans doute là que réside une cause des difficultés que rencontrent les élèves quand ils sont invités à passer, par un va-etvient permanent, du style rhétorique avec son relevé des traits formels et tout particulièrement les figures, au style herméneutique qui demande une synthèse et une interprétation plus globale (au niveau de l'œuvre) ${ }^{12}$.

31 Transposé, l'art des figures montre bien ce qui est requis des élèves : l'apprentissage d'une langue littéraire, car en réalité, même considérablement sédimentée, transformée, la rhétorique des figures promeut et fonde un "Ordre de la dignité littéraire » (Genette, 1966, p. 214). Comme la reconnaissance de la figure et sa littérarité dépendent essentiellement de la capacité du lecteur à distinguer le figuré du littéral, on se trouve effectivement en présence d'un facteur de distinction entre les élèves capables de verbaliser le littéraire par le métalangage requis et ceux qui n'y parviennent pas. Mais bien sûr, cette capacité renvoie en fin de compte à la forme que peut prendre la subjectivité dans le rapport aux textes : certaines formes de celle-ci étant valorisées, d'autres rejetées. La classe est ainsi le lieu où l'on régule selon des modalités fortement codées l'expression des émotions : pleurer à chaudes larmes la mort de Léopoldine suscitera un jugement négatif, en revanche être capable de 
caractériser la douleur paternelle par les procédés du lyrisme sera valorisé. On voit bien en ce cas que l'appréciation de la subjectivité interprétative dépend étroitement d'un assujettissement didactique, tel qu'il est opéré au travers d'un dispositif d'enseignement et d'apprentissage orienté par une sédimentation (Schneuwly et Dolz, 2009) de pratiques historiques.

Le style devient ainsi un élément privilégié de l'idéologie littéraire scolaire, mais d'une manière différente que pour les autres acteurs du champ littéraire, car, pris dans la logique de l'institution, il assujettit selon des modalités qui lui sont propres. Or, n'oublions pas que, pour les élèves, la verbalisation du style est sans doute l'une des tâches les plus ardues de l'explication de texte; elle requiert en effet une distance critique, un outillage conceptuel sophistiqué, une disciplination très contrôlée de l'émotion. Nous voici donc en présence d'une forme d'assujettissement imparable pour distinguer et primer un sujet didactique qui peut désormais être dit littéraire.

\section{Conclusion}

Résolument hors de portée d'une "science didactique expérimentale ( Brousseau, 2012, p. 101) dont les preuves seraient réfutables, le triple problème conceptuel du didactique, du littéraire et du sujet nous place devant la dimension fondamentalement sociohistorique de la didactique et de ses modélisations. Dans les contextes scolaires, pour expliquer les phénomènes qui lient l'émergence des sujets psychologiques à la culture et à ses idéologies, l'abstraction piagétienne du sujet épistémique, toute utile qu'elle soit dans l'espace de l'épistémologie génétique, n'est pas plus pertinente que ne le sont les conceptions personnalistes ou idéalistes du sujet. C'est bien par une prise en considération du sujet didactique que certains phénomènes caractéristiques du système didactique deviennent intelligibles.

De notre point de vue, sur un plan générique, le système didactique peut s'éclairer lorsque s'y reconnait une technique sociale - au sens vygotskien (1925/2005, p. 347) - de mise en relation et de transformation. Cette technique sociale a la particularité de prendre en charge d'autres techniques sociales spécifiant des mises en relation et des transformations particulières : ce sont ces manières de dire, de penser et d'agir qui, structurées en disciplines tout en demeurant évolutives, sont sélectionnées pour faire l'objet d'enseignements.

Alors que les dispositifs didactiques matérialisent de façon toujours singulière les relations systémiques entre les objets d'enseignement, les tâches et les activités des enseignants et des élèves, nous avons voulu montrer qu'y convergent les processus de transposition et d'assujettissement didactique. Il faut bien marquer que ces deux processus sont inséparables : toute activité humaine, avant même qu'elle soit retenue pour être enseignée et apprise, implique déjà un assujettissement spécifique - en littérature, comme dans le sport ou le génie civil. Dès lors, la construction des objets d'enseignement et d'apprentissage entraine inévitablement la transposition conjointe de savoirs et d'assujettissements. Entre possibilités et impossibilités pérennes ou provisoires, l'apprentissage requiert, autrement dit, de répondre à l'appel social de pouvoir être non pas un, mais une pluralité de sujets en fonction des situations d'interaction. On comprend mieux en ce sens que la didactique puisse apparaitre comme une science majeure pour la compréhension du développement de la personnalité1 ${ }^{13}$. Elle est ainsi amenée à jouer un rôle déterminant pour rendre compte de 
la dialectique entre l'histoire sociale et la biographie personnelle dans la mesure même où «La biographie [ainsi comprise] est à la personnalité ce que l'histoire est à la formation sociale : elle est l'histoire dans laquelle la personnalité, pour autant qu'elle y réussisse, se constitue, s'active, se transforme jusqu'à la fin » (Sève, 2008, p. 514).

\section{BIBLIOGRAPHIE}

ADORNO, T. W. (2016). Le Conflit des sociologies : théorie critique et sciences sociales. Paris : Payot.

ALTHUSSER, L. (2011). Sur la reproduction. Paris : PUF.

BALIBAR, E. (2005). « Préface ». In : Althusser L., Pour Marx. Paris : La Découverte.

BALIBAR, E., CASSIN, B. \& DE LIBERA, A. (2019). « Sujet ». In : Cassin, B. (dir.).Vocabulaire européen des philosophies : dictionnaire des intraduisibles. Paris : Le Seuil/Le Robert.

BRONCKART, J.-P. (2019). « Psychologue, psychopédagogue, linguiste et didacticien enfin : histoires d'un développement ». In : Aeby-Daghé, S., et al. (coord.), Didactique du français et construction d'une discipline scientifique. Dialogues avec Bernard Schneuwly. Villeneuve d'Ascq : Presses Universitaires du Septentrion, p. 25-37.

BROSSARD, M. (2008). «Concepts quotidiens/ concepts scientifiques : réflexions sur une hypothèse de travail ». Carrefours de l'éducation 26, p. 67-82. En ligne : https://doi.org/10.3917/cdle.026.0067. BROUSSEAU, G. (1998). Théorie des situations didactiques : Didactique des mathématiques 1970-1990. Grenoble : La pensée sauvage.

BRousSeAu, G. (2012). « Des dispositifs piagétiens... aux situations didactiques ». Éducation et Didactique, 6 (2), p. 103-129. En ligne : https://doi.org/10.4000/educationdidactique.1475.

CHEVAlLARD, Y. (1988). « Médiation et individuation didactiques ». Interactions didactiques, 8, p. 23-34. En ligne : http://yves.chevallard.free.fr/spip/spip/IMG/pdf/

Mediations_et_individuation_didactiques.pdf.

CHEVALLARD, Y. (1994). « Les processus de transposition didactique et leur théorisation ». In : Arsac, G., Chevallard, Y., Martinand, J.-L. \& Tiberghien, A. (dirs), La Transposition didactique à l'épreuve. Grenoble : La Pensée Sauvage, p. 135-180.

CHEVALLARD, Y. (1998). « Analyse des pratiques enseignantes et didactique des mathématiques: l'approche anthropologique ». Actes de l'UE de la Rochelle, p. 91-118. En ligne : http:// yves.chevallard.free.fr/spip/spip/IMG/pdf/Analyse_des_pratiques_enseignantes.pdf.

CHEVALLARD, Y. (2010). « Le sujet apprenant entre espace et dispositif. Commentaires depuis la théorie anthropologique du didactique ». Communication présentée aux journées du Lisec Gérardmer. En ligne : http://yves.chevallard.free.fr/spip/spip/IMG/pdf/ Commentaires_depuis_la_TAD_YC_.pdf. DAUNAY, B. (2007). « Le sujet lecteur : une question pour la didactique du français ». Le Français aujourd'hui 157, p. 43-51. En ligne : https://www.cairn.info/revue-le-francais-aujourd-hui-2007-2page-43.htm. 
DAUNAY, B. (2013). « Système didactique- Triangle didactique ». In : Reuter, Y., Cohen-Nazria, C., Daunay, B., Delcambre, I. \& Lahanier-Reuter, D. Dictionnaire des concepts fondamentaux des didactiques. Bruxelles : De Boeck, p. 203-210.

DAUnAY, B. (2014). « De l'imbécile en didactique du français ». In : Daunay, B. \& Dufays, J.-L. (dirs), Didactique du français : du côté des élèves. Comprendre les discours et les pratiques des apprenants. Louvain-la-Neuve : De Boeck, p. 175-185.

DAUNAY, B. (2016). « Quelques réflexions sur le sujet d'une description didactique ». Éducation et didactique, 10 (2), p. 123-136. En ligne : https://journals.openedition.org/educationdidactique/ 2488 .

DESCOMBES, v. (2004). Le Complément de sujet. Paris : Gallimard.

DUFAYS, J.-L. (2013). « Sujet lecteur et lecture littéraire : quelles modélisations pour quels enjeux ?», Recherches et travaux 83, p. 77-88. En ligne : https://doi.org/10.4000/recherchestravaux.640. FALLENBACHER-CLAVIEN, F. (2017). «Autours » des œuvres littéraires : les métatextes d'auteurs en classe de lycée. Thèse en sciences de l'éducation : université de Genève.

GABATHULER, C. (2016). Apprécier la littérature. La relation esthétique dans l'enseignement de la lecture de textes littéraires. Rennes : Presses universitaires de Rennes.

GABATHULER, C., VEDRINES, B. \& VUILLET, Y. (2019). « Didactique, réputation littéraire et connivences ». Recherches 70, p. 137-162.

GRIGNON, C. \& PASSERON, J.-C. (2015). Le Savant et le populaire. Paris : Seuil.

HACKING, I. (2008). Entre science et réalité. La construction sociale de quoi ? Paris : La Découverte.

HALTÉ, J.-F. (1992). La Didactique du français. Paris : PUF (collection Que-Sais-Je ?)

HALTÉ, J.-F. (2008). « Le français entre rénovation et reconfiguration ». Pratiques 137-138, p. 23-38.

En ligne : https://doi.org/10.4000/pratiques.1150.

HOFSTETTER, R. \& SCHNEUWLY, B. (dirs) (2007). Émergence des sciences de l'éducation à la croisée des traditions académiques contrastées. Fin du XIX ${ }^{e}$ - première moitié $d u X X^{e}$ siècle. Berne : Peter Lang. HOFSTETTER, R. \& SCHNEUWLY, B. (2014). « Disciplinarisation et disciplination consubstantiellement liées. Deux exemples prototypiques sous la loupe : les sciences de l'éducation et les didactiques des disciplines ». In : Engler, B. (éd.), Disziplin - Discipline. Fribourg : Academic Press, p. 27-46. KUHN, T. s. (1983). La Structure des révolutions scientifiques. Trad. de l'anglais (États-Unis). Paris : Flammarion.

LAURENT, J.-P. (2011). « Quelques enjeux didactiques d'une configuration de la discipline "français" ». In Daunay B., Reuter Y. \& Schneuwly B. (dirs), Les Concepts et les méthodes en didactique du français. Namur : Presses universitaires de Namur, p. 61-82.

LEBEAUME, J. (2011). « L'éducation technologique au collège : un enseignement pour questionner la refondation du curriculum et les réorientations des disciplines ». Éducation et didactique 5-2, p. 7-22. En ligne : https://doi.org/10.4000/educationdidactique.1178.

LIGOZAT, F., COQUIDÉ, M., MARLOT, C. VERSHEURE, I. \& SENSEVY, G. (2014). « Didactiques et/ou didactique. Poursuivre le travail de problématisation ». Éducation \& Didactique 8-1, p. 101-115. En ligne : http://journals.openedition.org/educationdidactique/1907. 
LOUICHON, B. (2011). « La lecture littéraire est-elle un concept didactique ? »In : Daunay, B., Reuter, Y. \& Schneuwly, B. (dirs), Les Concepts et les méthodes en didactique du français (195-216). Namur : Presses universitaires de Namur, p. 195-216.

MARTINAND, J.- L. (2016). « La question de la référence en didactique du curriculum ». Investigações em Ensino de Ciências, 8 (2), p. 125-130.

MARTINAND, J. - L. (2014). « Didactique et didactiques. Esquisse problématique ». In : Beillerot, J. \& Mosconi, N. (dir.).Traité des sciences et des pratiques de l'éducation. Paris : Dunod, p. 353-367. NOILLE-CLAUZADE, C. (2004). Le Style. Paris : Flammarion.

PASSERON, J.C. (1994). « De la pluralité théorique en sociologie : théorie de la connaissance sociologique et théories sociologiques ». Revue européenne des sciences sociales 32 (99), p. 71-116. PASSERON, J.-C. (2001). « La forme des preuves dans les sciences historiques ». Revue européenne des sciences sociales XXXIX-120, p. 31-76 En ligne : https://journals.openedition.org/ress/655.

PASSERON, J.-C. (2006). Le Raisonnement sociologique : un espace non poppérien de l'argumentation. Paris : Albin Michel.

POPPER, K. R. (1985) [1963]. Conjectures et réfutations : la croissance du savoir scientifique. Trad. de l'anglais (Grande-Bretagne) par M. I. et M. B. de Launay. Paris : Payot.

RENAUT, A. (2010). Découvrir la philosophie : le sujet. Paris : O. Jacob.

REUTER, Y., COHEN-AZRIA, C., DAUNAY, B., DELCAMBRE, I. \& LAHANIER-REUTER, D. (2013) Dictionnaire des concepts fondamentaux des didactiques. Bruxelles : De Boeck.

RONVEAUX, C. \& SCHNEUWLY, B. (2018). Lire des textes réputés littéraires : disciplination et sédimentation. Enquête au fil des degrés scolaires en Suisse romande. Berne : Peter Lang.

SCHNEUWLY, B. (1995). « De l'utilité de la "transposition didactique" ». In : Chiss, J.-L., David, J. \& Reuter, Y. (dirs), Didactique du français. État d'une discipline. Paris : Nathan, p. 47-62.

SCHNEUWLY, B. \& DOLZ, J. (2009). Des objets enseignés en classe de français. Rennes : Presses universitaires de Rennes.

SCHUBAUER-LEONI, L. (1997). «Entre théories du sujet et théories. Des conditions de possibilité du didactique : quel "cognitif" ? " Recherches en didactique des mathématiques, 17 (1), p. 7-27. En ligne : https://revue-rdm.com/1997/entre-theories-du-sujet-et/.

SENSEVY, G. \& LIGOZAT, F. (2017). « Didactique comparée et générale ». In : van Zanten, A. \& Rayou, P. (éds). Dictionnaire de l'éducation. Paris : Presses universitaires de France, p. 173-177

SENSEVY, G. \& MERCIER, A. (éds) (2007). Agir Ensemble. L'action didactique conjointe du professeur et des élèves dans la classe, Rennes : Presses universitaires de Rennes, 13-49.

SÈVE, L. (2008). Penser avec Marx aujourd'hui. Tome 2, L'homme ?. Paris : La Dispute.

SPITZER, L. (1970) [1928]. Études de style. Trad. de l'anglais (Grande-Bretagne) et de l'allemand par E. Kaufholz, A. Coulon \& M. Foucault. Paris : Gallimard.

VÉDRINES, B. (2017). L'Assujettissement littéraire. Thèse en sciences de l'éducation : université de Genève. En ligne : https://archive-ouverte.unige.ch/unige:102384/ATTACHMENT01. VÉDRINES, B. \& GABATHULER, C. (2018). « De la réputation "littéraire" ». In : Schneuwly, B. \& Ronveaux, C. (éds). Lire des textes réputés littéraires au fil des niveaux scolaires. Berne : Peter Lang, p. 67-81. 
VUILLET, Y. (2017). À la recherche didactique de concepts pour penser, dire et agir le littéraire. Thèse en sciences de l'éducation : université de Genève. En ligne : https://archive-ouverte.unige.ch/unige: 129260 .

VUILLET, Y. (2018). « Un poème entre réfractions littéraires et scolaires ». Pratiques 179/180. En ligne : https://journals.openedition.org/pratiques/5170.

VUILLET, Y. \& GABATHULER, C. (2017). « Approcher l'enseignement du littéraire comme essentiellement contestable ». In : Brunel, M., Émery-Bruneau, J., Dufays, J.-L., Dezutter, O. \& Falardeau, E. (éds), L'enseignement et l'apprentissage de la lecture aux différents niveaux de la scolarité. Namur : Presses universitaires de Namur, p. 51-77. En ligne : https://books.openedition.org/pun/ 5053?lang=fr.

VYGOTSKI, L. s. (2005) [1925]. La Psychologie de l'art. Trad. du russe par F. Sève. Paris : La Dispute. VYGOTSKI, L. S. (2014) [1931]. Histoire du développement des fonctions psychiques supérieures. Trad. du russe par F. Sève. Paris : La Dispute.

\section{NOTES}

1. En cela, de notre point de vue, la dimension sociohistorique de l'empirie didactique ordinaire s'oppose à l'inscription de la didactique dans l'espace épistémologique des sciences expérimentales.

2. Assumer la dimension interprétative des raisonnements didactiques ne doit pas être vu comme une négation, ni même comme une relativisation de leur scientificité. Pour synthétiser les précisions de J.-C. Passeron (2001, p. 44), nous pouvons reconnaitre avec lui que ces raisonnements sont scientifiques dans la mesure où 1) ils permettent des argumentations sémantiquement consistantes tout en demeurant 2) porteurs d'une vulnérabilité empirique lorsque formulés dans des protocoles d'enquêtes, et ce, pour autant que 3) le langage théorique employé permette d'énoncer une probabilité de cooccurrence ou de succession d'événements, et de la vérifier.

3. Nous précisons plus bas la teneur de ce concept.

4. Pour une problématisation d'ensemble de la configuration de la discipline "français ", nous proposons de se référer par exemple à J.-F. Halté (2008) et à J.P. Laurent (2011).

5. En pointant pertinemment qu'il n'est pas nécessaire de s'accorder théoriquement sur une définition « $d u$ » littéraire pour l'enseigner ou pour discourir à son sujet, il arrive que des doutes soient émis quant au fait que cette absence de définition fasse réellement problème en didactique. De notre point de vue, ces doutes accordent trop peu d'importance aux différences fonctionnelles qui permettent de distinguer les trois espaces didactiques de l'enseignement et de l'apprentissage, de la prescription et de la recherche. Pour décrire et expliquer un objet en tant qu'il est enseigné et appris, il nous semble pouvoir être attendu de la recherche en didactique du français qu'elle soit en mesure de le définir : renoncer à une telle définition reviendrait sinon à reproduire, sous le langage de descriptions scientifiques, ce qu'il s'agirait plutôt de décrire et d'expliquer. Sans nier la légitimité et la nécessité des orientations prescriptive et pragmatique en didactique du français, nous soulignons que la recherche doit aussi, sinon surtout, assurer un positionnement descriptif et critique vis-à-vis de ses objets. 
De ce point de vue, l'absence de définition «du» littéraire fait indéniablement problème.

6. Ce concept, dont on trouve une définition chez C. Ronveaux et B. Schneuwly (2018), permet d'entendre la littérature comme : "construction socio-historique, sans cesse redéfinie [...] à laquelle participent trois institutions : (i) le champ de la production de la "littérature" et sa critique, (ii) les études littéraires et (iii) l'école elle-même. Cette dernière produit de la "littérature" par des pratiques spécifiques d'études des textes dont elle assure la réputation littéraire, cette réputation étant le produit d'innombrables discours et pratiques, dont précisément ceux de l'école ». (p. 20)

7. Sans détailler une controverse alimentée par divers courants philosophiques aux $x^{e}$ et $\mathrm{XXI}^{\mathrm{e}}$ siècles, on peut retenir toutefois que l'humanisme qui « a consisté à valoriser en l'homme la double capacité d'être conscient de lui-même (l'autoréflexion) et de fonder son propre destin (l'autofondation)» a été profondément remis en question au point que parait désormais insoutenable «l'idée classique de subjectivité [...] désignant l'aptitude, où se situerait l'humanité de l'homme, à être l'auteur conscient et responsable de ses pensées et de ses actes "(Renaut, 2010, p.20). On peut aussi se reporter sur ce point à V. Descombes (2004) ou encore à l'article éclairant de E. Balibar, B. Laugier et A. De Libera (2019).

8. Pour une présentation des enjeux du système didactique, on peut se reporter à $B$. Daunay (2013).

9. À cet égard, même si nous ne partageons pas son positionnement théorique (ce que nous discuterons ailleurs), il faut reconnaitre au courant didactique du sujet lecteur le mérite d'avoir contribué à la question de la subjectivité et d'avoir ainsi suscité un débat. Cf. par exemple le numéro 157 du Français aujourd'hui (2007), et J.-L. Dufays (2013).

10. On peut se reporter pour une synthèse à l'entrée Elève - Apprenant - Sujet didactique (Reuter, 2013),

11. Nous renvoyons à l'analyse de B. Daunay sur l'imbécile en didactique (2014).

12. On peut se reporter pour des exemples de ces difficultés observées dans les interactions entre élèves et enseignants à B. Védrines (2017).

13. Nous la comprenons ici dans les termes de L.S. Vygotski, à savoir «le développement de cette synthèse psychique la plus haute qu'on doit avec juste raison appeler la personnalité de l'enfant. L'histoire du développement culturel pendant l'enfance nous conduit à l'histoire du développement de la personnalité » (1931/2014, p. 137). M. Brossard commente ainsi cette définition: «[L.S. Vygotski] ne prend le concept de personnalité ni dans le sens courant du terme ni même dans le sens où l'emploie habituellement la psychologie différentielle contemporaine. Il prend ce terme dans un sens développemental : il s'agit de la structure d'ensemble qui se construit de l'enfance à l'adolescence et qui rassemble en une totalité les différentes fonctions du système psychique humain. Le concept de personnalité est étroitement lié à celui de conception du monde. Comme dans la théorie piagétienne - mais avec cette différence essentielle qu'ici les contenus jouent un rôle décisif -, nous avons affaire à une construction corrélative : en même temps que se construit un système psychologique, le sujet construit une conception du monde physique, social et psychologique. On voit que les connaissances occupent une place centrale dans le développement de la personnalité » $(2008$, p. 81). 


\section{RÉSUMÉS}

Notre article, de nature théorique, s'intéresse au triple problème conceptuel du didactique, du littéraire et du sujet. Parce que les référents auxquels renvoient ces trois termes sont pluriels et discutables, nous approchons dans un premier temps la didactique, ses modèles, en tant qu'ils relèvent de l'espace sémantique d'une science sociohistorique. Nous abordons ensuite les implications, pour la didactique $d u$ français, de ce triple problème. Ceci nous conduit à argumenter en faveur du développement d'une théorie du «sujet didactique " pour traiter de l'enseignement et de l'apprentissage de «la» littérature. En décrivant des processus d'assujettissement induits par divers types de dispositifs, nous abordons finalement le problème du lien entre le psychisme individuel et les rapports sociaux, et proposons ainsi de réfléchir à la question de l'individualité et de ses formes historiques.

Our article, theorical in nature, deals with the threefold conceptual problem of didactics, literature and subject. Because the referents to which these three terms refer are plural and debatable, we first approach didactics, its models, as they belong to the semantic space of a sociohistorical science. Then we discuss the implications of this triple problem for the didactics of French. This leads us to argue in favour of developing a theory of the "didactic subject" to approach the teaching and learning of "the" literature. Finally, by describing processes of subjection induced by various types of devices, we address the problem of the link between the individual psyche and social relationships, and thus propose to reflect upon the question of individuality and its historical forms.

\section{INDEX}

Keywords : didactic(s), literary reputation, subjection, didactic subject, didactic system Mots-clés : didactique(s), réputation littéraire, assujettissement, sujet didactique, système didactique.

\section{AUTEURS}

\section{YANN VUILLET}

Haute École Pédagogique du Valais, CH-1890, Saint-Maurice, Suisse

\section{BRUNO VÉDRINES}

Université de Genève, $\mathrm{CH}-1211$, Genève, Suisse 